\title{
Sugammadex: a turning point in our practice
}

\author{
Sangseok Lee \\ Department of Anesthesiology and Pain Medicine, Sanggye Paik Hospital, College of Medicine, Inje University, Seoul, Korea
}

Curare, which is the source of modern muscle relaxants, was used for centuries by South American Indians for hunting. On January 23, 1942, Harold R. Griffith and a resident Enid Johnson administered curare (intocostrin) to a patient undergoing an appendectomy, and this was the first clinical use of curare [1]. After World War II, Dr. Cecil Gray and John Halton used the drug on patients as a balanced anesthesia technique known as the Liverpool technique, a triad of narcosis, analgesia, and muscle relaxation, which is the essential anesthetic technique in use today [2]. Succinylcholine was developed by Thesleff and Foldes in 1952 and has changed anesthetic practice dramatically. Succinylcholine has a unique rapid onset and ultra-short duration of action. It facilitates rapid endotracheal intubation and rapid recovery from relaxation and is a widely used drug despite several side effects.

Savarese and Kitz [3] reported that the ideal muscular blocking agent should have the following characteristics: 1) a brief, non-cumulative, non-depolarizing action; 2) rapid onset and recovery; 3) high potency and selectivity; 4) lack of active metabolites; 5) reversible by an appropriate antagonist; and 6) lack of clinically important cardiovascular (autonomic, hemodynamic) side effects. Many scientists have been trying to identify an ideal ultra-short acting non-depolarizing muscle relaxant as a replacement for succinylcholine, but this has not been achieved.

Scientists hold the view that an alternative approach is now needed, after considerable time and expense has been put into the replacement project, without result. They were of the opinion that a fast-onset and medium-duration action relaxant that is readily reversible, rather than a fast-onset and short-duration action relaxant, would be the best alternative to succinylcholine. Finally, their hopes have been realized, because the combination of rocuronium or vecuronium and sugammadex aligns very well to their modified concept of the ideal muscle relaxant.

In this issue of the Korean Journal of Anesthesiology, Professor Fuchs-Buder et al. [4] summarizes the development and clinical use of sugammadex. The authors broadly review pharmacologic properties, which show the reliability of sugammadex, as well as its side effects. They indicate that sugammadex should help increase therapeutic options. I agree with the authors' view in the review article. We can freely and precisely control the depth of muscle relaxation using sugammadex during surgery. I have no doubt that our clinical practice will be greatly influenced by sugammadex hereafter.

However, as sugammadex is a relatively new drug, there are an insufficient number of studies on a variety of populations. The manufacturer recommends special caution using sugammadex in patients with highly depressed hepatic or renal function, infants, and in obstetric cases [5]. Hypersensitivity, which has caused a delay in FDA approval in the United States, is also a concern, as well as other adverse effects [6]. A randomized, safety assessor-blinded trial that included 128 Korean patients undergoing general anesthesia has been conducted. In this issue of the Korean Journal of Anesthesiology, Woo et al. [7] shows that sugammadex provides 8.1 times faster recovery from moderate rocuronium-induced neuromuscular block than neostigmine in Korean patients. The authors report that sugammadex was generally well tolerated, with no evidence of residual or recurrent neuromuscular block. The overall efficacy and safety reports for sugammadex were similar to those of Caucasian patients in a comparable pivotal study that examined reversal of moderate neuromuscular block [8].

However, sugammadex is not an omnipotent drug, and one

Corresponding author: Sangseok Lee, M.D., Department of Anesthesiology and Pain Medicine, Sanggye Paik Hospital, College of Medicine, Inje University, 761-1, Sanggye7-dong, Nowon-gu, Seoul 139-707, Korea. Tel: 82-2-950-1171, Fax: 82-2-950-1323, E-mail: sslee@paik.ac.kr (c) This is an open-access article distributed under the terms of the Creative Commons Attribution Non-Commercial License (http:// creativecommons.org/licenses/by-nc/3.0/), which permits unrestricted non-commercial use, distribution, and reproduction in any medium, provided the original work is properly cited. 
drug cannot change long-standing practice. I always recommend monitoring the depth of neuromuscular block and its reversal routinely in daily clinical practice. About $20 \%$ of patients arrive in the post-anesthesia care unit with train-of-four (TOF) ratios $<0.70$, and $40 \%$ arrive with TOF values $<0.90$ after administration of a vecuronium or atracurium [9]. Furthermore, a shortacting neuromuscular block such as mivacurium may cause residual postoperative weakness [10]. Seo et al. [11] reported that $83.3 \%$ of anesthesiologists in Korea still prefer to use clinical signs rather than quantitative neuromuscular monitoring as an evaluation method for residual neuromuscular blockade. The basic principle of reversing sugammadex dosing is based on the depth of the neuromuscular block. The manufacturer recommends that the dose should be $4 \mathrm{mg} / \mathrm{kg}$ for a deep block (two post-tetanic responses), $2 \mathrm{mg} /$ for a moderate block (T2 response of TOF stimulation), and $16 \mathrm{mg} / \mathrm{kg}$ for immediate (rescue) reversal after a large dose of rocuronium [12]. Therefore, without information about the depth of neuromuscular block, an incorrect dose of sugammadex could be administered, which could cause the residual block or recurarization even after using sugammadex [13].

There is also an economic barrier to the wide introduction of sugammadex in Korea. Because of the low cost of medical treatment and highly restrictive governmental regulation policy, anesthesiologists in Korea are very sensitive to medical care costs. Therefore, further clinical trials that consider cost-effectiveness should be conducted.

We are ready for a turning point in our practice. Are you ready to adopt the new clinical challenge involved?

\section{References}

1. Griffith HR, Johnson GE. The use of curare in general anesthesia. Anesthesiology 1942; 3: 418-20.

2. Gray TC, Halton J. Technique for the use of ditubocurarine chloride with balanced anaesthesia. Br Med J 1946; 2: 293-5.

3. Savarese JJ, Kitz RJ. Does clinical anesthesia need new neuromuscular blocking agents? Anesthesiology 1975; 42: $236-9$.

4. Fuchs-Buder T, Meistelman C, Raft J. Sugammadex: clinical development and practical use. Korean J Anesthesiol 2013; 65: 495-500.

5. Bridion (Sugammadex) data sheet. Available from http://custom.kims.co.kr/DrugSearch/PopDrugGenMonoPrint.aspx?KIMSCode= EMSDSIJC5U3. Accessed November 1, 2013.

6. Godai K, Hasegawa-Moriyama M, Kuniyoshi T, Kakoi T, Ikoma K, Isowaki S, et al. Three cases of suspected sugammadex-induced hypersensitivity reactions. Br J Anaesth 2012; 109: 216-8.

7. Woo T, Kim KS, Shim YH, Kim MK, Yoon SM, Lim YJ, et al. Sugammadex versus neostigmine reversal of moderate rocuronium-induced neuromuscular blockade in Korean patients. Korean J Anesthesiol 2013; 65: 501-7.

8. Blobner M, Eriksson LI, Scholz J, Motsch J, Della Rocca G, Prins ME. Reversal of rocuronium-induced neuromuscular blockade with sugammadex compared with neostigmine during sevoflurane anaesthesia: results of a randomized, controlled trial. Eur J Anaesth 2010; 27: 874-81.

9. Fawcett WJ, Dash A, Francis GA, Liban JB, Cashman JN. Recovery from neuromuscular blockade: residual curarisation following atracurium or vecuronium by bolus dosing or infusions. Acta Anaesthesiol Scand 1995; 39: 288-93.

10. Bevan DR, Kahwaji R, Ansermino JM, Reimer E, Smith MF, O'Connor GA, et al. Residual block after mivacurium with or without edrophonium reversal in adults and children. Anesthesiology 1996; 84: 362-7.

11. Seo HJ, Lee YK, Lee SS, Kim KS, Yang HS. A survey of postoperative residual neuromuscular block and neuromuscular monitoring. Anesth Pain Med 2010; 5: 70-4.

12. Groudine SB, Soto R, Lien C, Drover D, Roberts K. A randomized, dose-finding, phase II study of the selective relaxant binding drug, sugammadex, capable of safely reversing profound rocuronium-induced neuromuscular block. Anesth Analg 2007; 104: 555-62.

13. Kotake Y, Ochiai R, Suzuki T, Ogawa S, Takagi S, Ozaki M, et al. Reversal with sugammadex in the absence of monitoring did not preclude residual neuromuscular block. Anesth Analg 2013; 117: 345-51. 